\title{
MicroRNA-373 promotes cell migration via targeting salt-inducible kinase 1 expression in melanoma
}

\author{
$\mathrm{XINPING} \mathrm{BAI}^{1}, \mathrm{MING} \mathrm{YANG}^{1}$ and $\mathrm{YI} \mathrm{XU}^{2}$ \\ ${ }^{1}$ Department of Plastic Surgery, Central Hospital of Wuhan, Tongji Medical College, \\ Huazhong University of Science and Technology, Wuhan, Hubei 430014; ${ }^{2}$ Department of Plastic Surgery, \\ The Third People's Hospital of Hubei Province, Wuhan, Hubei 430033, P.R. China
}

Received May 10,2017; Accepted September 13, 2017

DOI: $10.3892 / \mathrm{etm} .2018 .6784$

\begin{abstract}
It is well established that altered expression of microRNAs (miRs) is critical in numerous human cancer types. Nevertheless, the molecular mechanisms of many miRs are yet to be elucidated. In the present study, reverse transcription-quantitative polymerase chain reaction and western blot analyses, and cell migration assays were performed to verify dysregulation of miR-373 in melanoma and its biological function. The transcriptional level of miR-373 was identified to be upregulated in melanoma tissues and cell lines compared with nevus and normal melanocytes. miR-373 was identified to function as an oncomiR, promoting melanoma cell migration. Notably, miR-373 was observed to suppress its downstream gene salt-inducible kinase 1 (SIK1) through directly binding the 3'-untranslated region of SIK1 expression. Furthermore, reduced SIK1 expression was identified to be responsible for the oncogenic effect of miR-373. In conclusion, the present study indicates that miR-373 functions as an oncomiR to promote melanoma progression through targeting SIK1 expression. This may provide a new therapeutic approach for melanoma.
\end{abstract}

\section{Introduction}

The past decade has seen a rapid increase in the incidence in melanoma, it is estimated that the annual increase in the incidence rate of melanoma has been $\sim 3-7 \%$ per year worldwide for Caucasians (1). Melanoma is one of the most lethal of all adult malignancies due to its extremely high tendency to metastasize towards multiple human organs, such as the liver, brain and lung $(2,3)$. The median survival following the onset of distant metastases is 6-9 months, and the 5-year survival

Correspondence to: Dr Yi Xu, Department of Plastic Surgery, The Third People's Hospital of Hubei Province, 26 Zhongshan Road, Wuhan, Hubei 430033, P.R. China

E-mail: xydr0420@163.com

Key words: microRNA-373, migration, invasion, salt-inducible kinase 1, melanoma rate is $>5 \%(2,3)$. Surgery resection remains the primary curative treatment for melanoma at early stages (4). However, treatment of melanoma at late stages is difficult and less effective (5). Therefore, the development of new molecular targeted therapies for melanoma is urgently required.

MicroRNAs (miRs) are small non-coding RNAs (22-26 nucleotides), which inhibit the expression of target genes primarily by binding to the 3'-untranslated region (3'-UTR) of mature mRNAs, leading to mRNA degradation or inhibition of translation (6,7). Aberrant expression of miRs has been reported in various human cancer types. miRs have been reported to function as oncogenes or tumor suppressor genes in tumor progression as well as development, encompassing apoptosis, proliferation, migration, invasion, metastasis and resistance to therapy (7-10). Notably, numerous miRs serve pivotal roles in melanoma development and progression (11-13). miR-373 is among the most commonly upregulated miRs in melanoma (14). However, the detailed mechanism of miR-373 in melanoma is yet to be elucidated.

The serine/threonine kinase salt-inducible kinase 1 (SIK1) is a member of the SIK family, which consists of SIK1, SIK2 and SIK3 (15). SIK1 has been reported to be involved in multiple biological processes, including regulation of PKA activity (16) and p53-dependent anoikis through the use of a kinome-wide loss-of-function screen (17). Attenuated SIK1 gene expression is identified to promote various human cancer types. In non-small cell lung cancer, downregulation of SIK1 enhances epithelial-mesenchymal transition (EMT) and radioresistance (18). In addition, induced SIK1 expression has been reported to inhibit gastric cancer cell migration (19). However, the functional role of SIK1 in melanoma remains largely unknown.

In this study, to clarify the vital role of miR-373 in human melanoma, reverse transcription-quantitative polymerase chain reaction (RT-qPCR) and western blot analyses were used to detect the expression of miR-373 in clinical samples, wound healing assays were used to detect cell migration and Transwell assays were also used to detect cell migration. Based on the results of the current study, miR-373 was demonstrated to serve as an oncomiR in melanoma, by enhancing melanoma cell migration. Furthermore, miR-373 was observed to attenuate SIK1 protein level through directly binding its 3'-UTR. 
Notably, it was also identified that downregulation of SIK1 enhanced melanoma cell migration.

\section{Materials and methods}

Clinical sample collection. A total of 16 melanoma tissues and normal skin samples were collected from patients recruited from the Department of Plastic Surgery, Central Hospital of Wuhan, Tongji Medical College, Huazhong University of Science and Technology (Wuhan, China) between February 2015 and June 2016. The mean age of the recruited patients (10 male and 6 female) were 56.46 years (standard deviation, 2.63 years). The fresh tissues were frozen in liquid nitrogen $\left(-196^{\circ} \mathrm{C}\right)$ to protect the protein or RNA from degradation. The use of human tissues was approved by the Ethics Committee of the Central Hospital of Wuhan and all patients provided written, informed consent.

Cell culture and transfection. The human melanoma cell lines A375, WM115 and WM75 were purchased from the Shanghai Institute of Biochemistry and Cell Biology (Shanghai, China), and were maintained in Dulbecco's modified Eagle's medium (DMEM; Invitrogen; Thermo Fisher Scientific, Inc., Waltham, MA, USA) supplemented with $10 \%$ (v/v) fetal bovine serum (FBS; Thermo Fisher Scientific, Inc.), penicillin (25 U/ml), streptomycin $(25 \mathrm{~g} / \mathrm{ml})$ and $1 \% \mathrm{~L}$-glutamine. Normal human melanocytes were purchased from Lifeline Cell Technology, LLC (Frederick, MD, USA; cat. no. FC-0030) and grown in LL-0027 medium (Lifeline Cell Technology, LLC). All cell lines were cultured in a $5 \% \mathrm{CO}_{2}$ and $37^{\circ} \mathrm{C}$ incubator. For the upregulation of miR-373 expression, a synthesized miR-373 mimic (5'-GAAGUGCUUCGAUUUUGGGGUGU-3') and a miR-NC (5'-UUCUCCGAACGUGUCACGUTT-3' (both 10 nM; Shanghai GenePharma Co., Ltd., Shanghai, China) was transfected into A375 or WM115 cells using Lipofectamine 2000 (Invitrogen; Thermo Fisher Scientific, Inc.), according to the manufacturer's protocol. The medium containing the transfection reagents was removed $6 \mathrm{~h}$ after transfection and $48 \mathrm{~h}$ later the cells were harvested for subsequent assays. The transfection efficiency and changes in miR-373 expression were determined by RT-qPCR. For the downregulation of SIK1 expression, short hairpin (sh)RNA against SIK1 (sh-SIK1; Shanghai GenePharma Co., Ltd.) was stably transfected in A375 or WM115 cells using Lipofectamine 2000, according to the manufacturer's protocol. The sh-SIK1 sequence was 5'-UGA ACAAGAUCAAAGGGUU-3' and negative control shRNA (sh-NC) sequence was 5'-AATTCTCCGAACGTGTCACGT-3' (Hanyin Biotechnology, Shanghai, China). The transfection efficiency and changes in SIK1 expression were determined by western blotting. The medium containing the transfection reagents was removed $6 \mathrm{~h}$ after transfection and $48 \mathrm{~h}$ later the cells were harvested for the following assays.

Bioinformatics analysis. To determine whether overexpression of miR-373 promoted melanoma through targeting of the 3'-UTR of its downstream gene, three different algorithms [TargetScan (http://www.targetscan.org/vert_61/), miRTarBase (http://mirtarbase.mbc.nctu.edu.tw/php/index.php) and miRDB (http://mirdb.org/miRDB/)] were used to select the potential target genes of miR-373.
Wound healing assay. A375 or WM115 cells were cultured in a $25 \mathrm{~cm}^{2}$ culture flask to $80-90 \%$ confluence in DMEM supplemented with $10 \% \mathrm{FBS}$ at $37^{\circ} \mathrm{C}$, collected by digestion with $0.25 \%$ trypsin (Gibco; Thermo Fisher Scientific, Inc.) and centrifuged at $4^{\circ} \mathrm{C}$ at $1,409 \times \mathrm{g}$ for $5 \mathrm{~min}$, and then seeded into 6-well plates at $2.5 \times 10^{5}$ cells/well. miR-373 mimic, miR-NC, sh-SIK1 and sh-NC were transfected into A375 or WM115 cells as described above. Streaks were created in the monolayer with a pipette tip. Progression of migration was observed and photographed $24 \mathrm{~h}$ after wounding. The floating cells were removed by washing with $\mathrm{PBS}$, and the width of scratch was observed at 0 and $24 \mathrm{~h}$ using an inverted microscope (magnification, x50). Each experiment was repeated three times.

Transwell migration assay. The migration capability of A375 or WM115 cells was determined using a Transwell assay. The two cell lines were harvested and seeded with serum-free DMEM into the upper chambers at $5 \times 10^{4}$ cells/well. The bottom chambers contained DMEM with 10\% FBS. The Transwells were incubated for $24 \mathrm{~h}$ at $37^{\circ} \mathrm{C}$. Following incubation, the migrated cells attached to the lower surface of the membrane were fixed by $4 \%$ paraformaldehyde for $30 \mathrm{~min}$ at room temperature and stained with $1 \%$ toluidine blue for $15 \mathrm{~min}$ at room temperature. Cell numbers were counted in five randomly chosen fields using an inverted microscope (magnification, x100) per membrane and counts were repeated three times.

$R T-q P C R$. Total RNA from tissues or cells was extracted using TRIzol reagent (Invitrogen; Thermo Fisher Scientific, Inc.), according to the manufacturer's protocol. cDNA was synthesized using a PrimeScript RT reagent kit (Takara Bio, Inc., Otsu, Japan), according to the manufacturer's protocol. qPCR was performed with a KAPA SYBR FAST qPCR kit (Kapa Biosystems, Inc., Wilmington, MA, USA) using a 7900HT Fast Real-Time PCR system (Applied Biosystems; Thermo Fisher Scientific, Inc.). The initial denaturation step was for $10 \mathrm{sec}$ at $95^{\circ} \mathrm{C}$, followed by 40 cycles of denaturation for $5 \mathrm{sec}$ at $95^{\circ} \mathrm{C}$, and annealing and extension for $20 \mathrm{sec}$ at $60^{\circ} \mathrm{C}$. The expression levels of miRNA were normalized to endogenous small nuclear RNA U6. The $2^{-\Delta \Delta \mathrm{Cq}}$ method was used to analyze the expression level relative to the endogenous control (20). The primers used were as follows: miR-373 forward, 5'-ATT TTGGTTAATACGGTGAAATTTC-3' and reverse, 5'-CTA TCGCCCAAACTAAAATACGAT-3'; SIK1 forward, 5'-TGG ACGTCTGGAGCCTCGGT-3' and reverse, 5'-CTCGCGTTT TTCCTTAGCTG-3'; U6, forward, 5'-CTCGCTTCGGCA GCACA-3' and reverse, 5'-ACGCTTCACGAATTTGCGT-3'.

Western blot analysis. Protein extraction and western blotting analysis were conducted as described previously (21). The cells were incubated with the primary antibodies, including anti-SIK1 (cat. no. ab64428; 1:1,000; Abcam, Cambridge, MA, USA) or anti-GAPDH (cat. no. sc-25778; 1:5,000; Santa Cruz Biotechnology, Inc., Dallas, TX, USA). Then the membranes were then washed and incubated with horseradish peroxidase-conjugated goat anti-rabbit IgG secondary antibody (cat. no. sc-2004; 1:5,000; Santa Cruz Biotechnology, Inc.).

Vector construction and luciferase reporter assay. For the luciferase reporter assay, A375 cells were seeded on 24-well plates 
and co-transfected using Lipofectamine 2000 with $100 \mathrm{ng} /$ well firefly luciferase UTR-reporter vector, $2 \mathrm{ng} /$ well Renilla pRLCMV vector (internal control; all Promega Corporation, Madison, WI, USA) and $20 \mathrm{ng} /$ well of miR-373 mimics or miR-NC (Applied Biosystems; Thermo Fisher Scientific, Inc.), following the manufacturer's protocol. In brief, luciferase reporters were constructed through cloning of the 3'-UTR of SIK1 wild-type (wt) as well as SIK1 mutant-type (mut) via a deletion at position 1,905-1,911. The 3'UTR of SIK1 mRNA containing the wild-type or mutant miR-373 recognition sequences were PCR-amplified and subcloned into the SacI and SalI sites of the pmirGLO vector (Promega Corporation). A375 cells transfected with miR-373 or miR-NC and cultured in 48-well plates were co-transfected with $1.5 \mu \mathrm{g}$ of firefly luciferase reporter and $0.35 \mathrm{ng}$ Renilla luciferase reporter with Lipofectamine 2000 regent. After 24 h the relative luciferase activity was assessed with the Dual-Luciferase Assay Reporter system (cat. no. E1910; Promega Corporation) and normalized to Renilla luciferase activity.

Statistical analysis. Data are presented as the mean \pm standard deviation from at least three independent experiments. All data were analyzed using GraphPad 6.0 statistical software (GraphPad Software, Inc., La Jolla, CA, USA). Differences between two groups were analyzed using two-independent-sample Student's t-test or non-parametric Mann-Whitney U test. Differences between multiple groups were analyzed using one-way analysis of variance with Dunnett's post hoc test. $\mathrm{P}<0.05$ was considered to indicate a statistically significant difference.

\section{Results}

miR-373 is upregulated in melanoma tissues and cell lines. To investigate the expression of miR-373 in melanoma, RT-qPCR was performed. The results indicated that the mRNA level of miR-373 was significantly upregulated in melanoma tissues compared with nevus (Fig. 1A). Next, the expression of miR-373 was evaluated in a panel of normal melanocytes and human melanoma cell lines. The results indicated that miR-373 was significantly upregulated in A375, WM115 and WM75 melanoma cell lines compared with normal melanocytes (Fig. 1B). The results indicated that the expression of miR-373 is increased in melanoma tissues and cell lines.

miR-373 promotes melanoma cell migration. To explore the functional role of miR-373 in melanoma progression, melanoma cells [A375 (relatively highest level of miR-373 among the three cell groups from Fig. 1B) and WM115 (relatively lowest level of miR-373 among the three cell groups from Fig. 1B)] were stably transfected with miR-373 mimics. It was confirmed that the expression of miR-373 was upregulated in A375 and WM115 cells following miR-373 mimic transfection compared with cells transfected with miR-NC (Fig. 2A). As indicated in Fig. 2B and C, a wound healing assay demonstrated that transfection with miR-373 mimic significantly enhanced melanoma cell migration in A375 and WM115 cells compared with transfection with miR-NC. The effects of miR-373 on melanoma cell migration were evaluated further using a Transwell assay (Fig. 2D and E). A375 and WM115
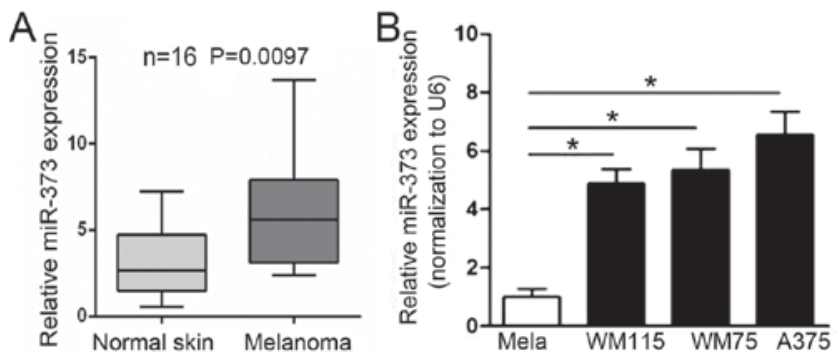

Figure 1. miR-373 is upregulated in melanoma tissues and cell lines. (A) Comparison of miR-373 expression in 16 melanoma tissues and normal skin tissues by reverse transcription-quantitative polymerase chain reaction. U6 was used as an internal control. (B) Comparison of miR-373 expression in a panel of human melanoma cell lines (A375, WM115 and WM75) and normal melanocytes. ${ }^{*} \mathrm{P}<0.05$ vs. Mela. Mela, normal melanocytes; miR, microRNA.

cells transfected with miR-373 mimic were observed to have a significantly higher migration capability compared with cells transfected with miR-NC. These results suggested that miR-373 elevates melanoma cell migration.

3'-UTR of SIK1 is the direct target of miR-373. Among the potential candidate targets of miR-373 was SIK1 (Fig. 3A). Fig. 3B indicates that the seed sequence of miR-373 is complementary to the 3'-UTR of SIK1 and is highly conserved among 18 different species. Dual-luciferase reporter assays illustrated that the transient transfection of A375 cells with SIK1 wt 3'-UTR significantly reduced reporter expression when compared with the control vector (Fig. 3C and D). However, the firefly luciferase reporter activity of the SIK1 mut 3'-UTR was not notably affected by miR-373-transfection (Fig. 3D). This result indicated that the 3'-UTR of SIK1 was targeted by miR-373. Furthermore, western blot analysis results demonstrated that overexpression of miR-373 inhibited SIK1 protein level in A375 and WM115 cells (Fig. 3E). These results supported the conclusion that SIK1 is the target gene of miR-373 in melanoma.

sh-SIK1 augments cell migration in melanoma. The functional role of SIK1 in melanoma was evaluated. The effect of sh-SIK1 and sh-NC on SIK1 expression in A375 and WM115 cell lines was observed via western blot analysis. It was confirmed that transfection with sh-SIK1 resulted in decreased SIK1 expression compared with sh-NC in the two cell lines (Fig. 4A). A375 cells and WM115 cells transfected with sh-SIK1 exhibited significantly increased cell migration compared with sh-NC Transfected cells, as demonstrated by wound healing and Transwell assays (Fig. 4B-E). These results indicated that downregulation of SIK1 promotes melanoma cell progression.

\section{Discussion}

Accumulating evidence indicates that miR-373 acts as a tumor suppressor or oncogene in numerous human cancer types (22). More specifically, miR-373 targeting CD44 and transforming growth factor- $\beta$ receptor 2 suppressed tumor migration and invasion in glioma cells (23). In parallel, miR-373 inhibited ovarian cancer cell invasion and metastasis by targeting RAB22A (24). On the other hand, miR-373 
A

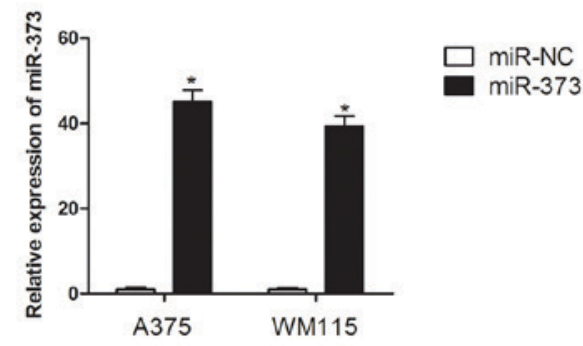

B
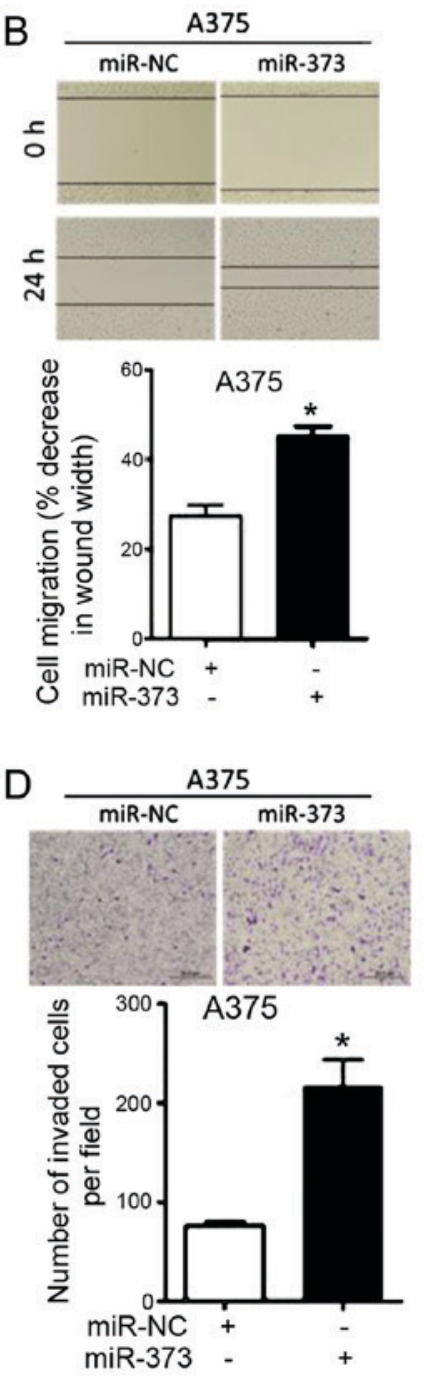

C
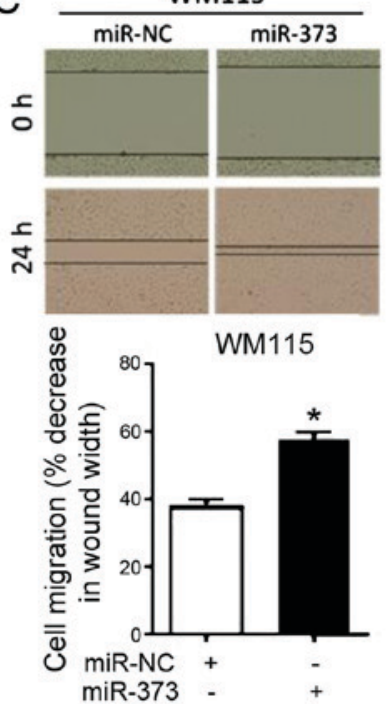

E
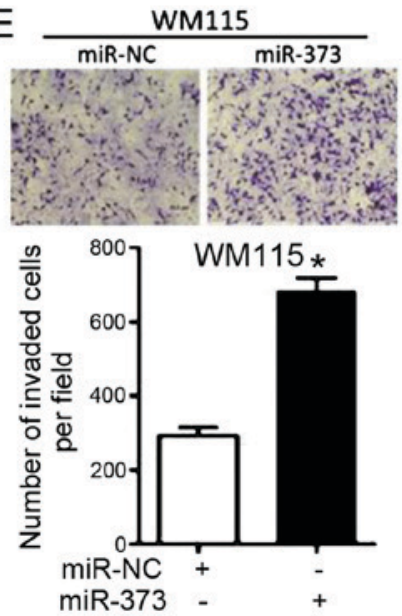

Figure 2. miR-373 promotes melanoma cell migration. (A) qRT-PCR assays for the miR-373 and miR-NC levels in A375 and WM115 cells demonstrated the transfection efficiency. A wound healing assay was performed after transfection with miR-373 or miR-NC in (B) A375 and (C) WM115 melanoma cells. A Transwell migration assay was performed after transfection with miR-373 or miR-NC in (D) A375 and (E) WM115 melanoma cells. "P $<0.05$ vs. miR-NC. miR, microRNA; miR-373, miR-373 mimic; miR-NC, negative control miR. Magnification, $\mathrm{x} 100$.

was associated with aggressive human mucinous colorectal cancer (25). Upregulated miR-373 promoted cell migration in breast cancer through epigenetic silencing of integrin subunit $\alpha-2$ (26). miR-373 enhanced EMT transition and metastasis via targeting thioredoxin-interacting protein in breast cancer (27). However, the association between miR-373 and melanoma remains unclear.

In the present study, it was demonstrated that the expression of miR-373 is upregulated in melanoma tissues and

A

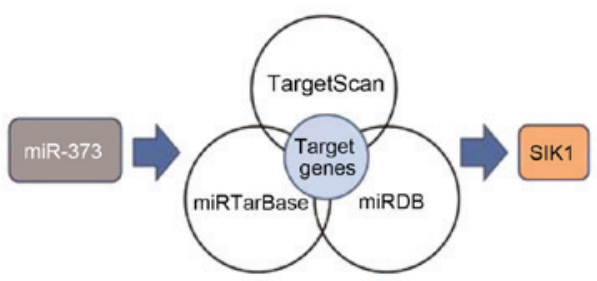

B

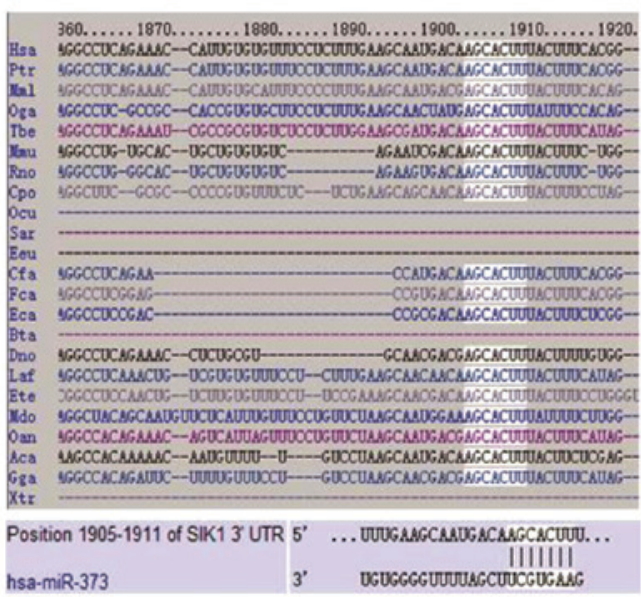

C

miRNA-373 3' ugugggguuuuagcuUCGUGAAg 5'

SIK1 3'UTR wt 5' uugaagcaaugacaAGCACUUu 3'

SIK1 3'UTR mut 5' uuugaagcaaugaca---u 3'
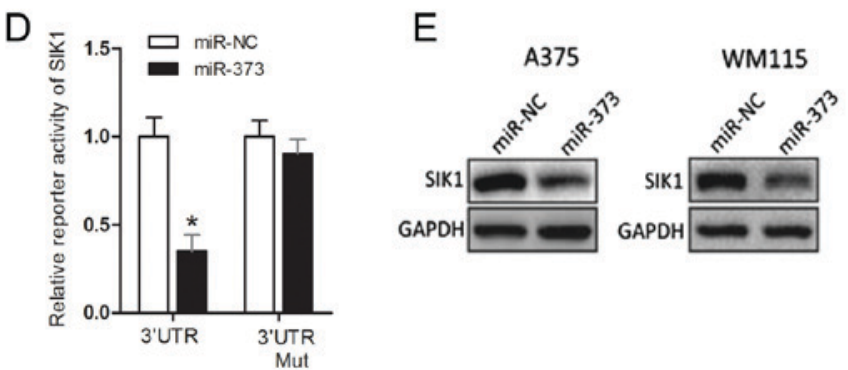

Figure 3. SIK1 3'-UTR is the direct target of miR-373. (A) Schematic illustration of the proposed model, depicting SIK1 as the potential target gene from three miR prediction databases: TargetScan, miRTarBase and miRDB. (B) The miR-373 seed sequence is complementary to the 3'-UTR of SIK1 and is conserved in 18 different species. (C) Predicted binding site of miR-373 in the 3'-UTR of SIK1, with sequences of the SIK1 3'-UTR wt and SIK1 3'-UTR mut. (D) SIK1 3'-UTR luciferase reporter assays in A375 cells. (E) Western blot analysis of SIK1 protein levels in A375 and WM115 cells after transfection with miR-NC or miR-373 mimic. "P<0.05 vs. miR-NC. SIK1, salt-inducible kinase 1; miR, microRNA; 3'-UTR, 3'-untranslated region; wt, wild-type; mut, mutated; $\mathrm{NC}$, negative control.

certain melanoma cell lines compared with nevus and normal melanocytes, respectively. Functionally, miR-373 mimics significantly enhanced cell migration in wound healing and Transwell assays in A375 and WM115 cell lines. miR-373 was identified to target downstream gene SIK1 through directly binding a complementary sequence. In addition, downregulated SIK1 was indicated to be promote migration in A375 and WM115 cell lines. Collectively, these findings suggest that miR-373 serves as an oncomiR to promote melanoma progression and development via targeting SIK1.

Previous studies have illustrated that SIK1 is downregulated in multiple human tumor types. In human hepatocellular carcinoma (HCC), SIK1 markedly inhibited 


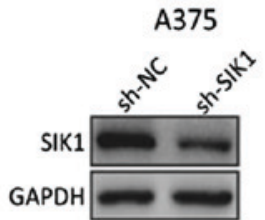

B
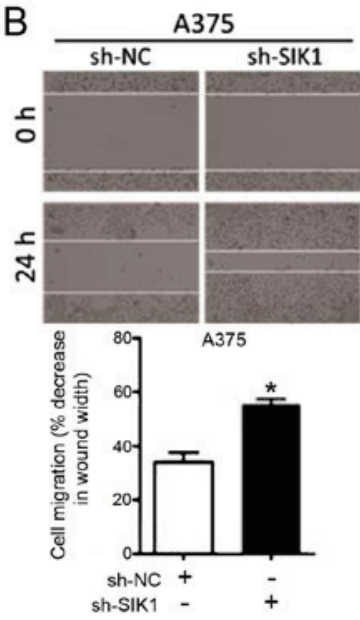

D
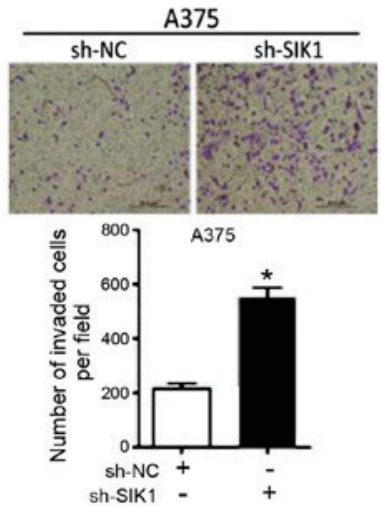

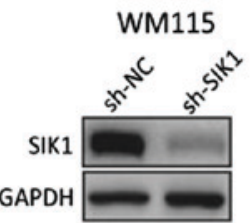

C
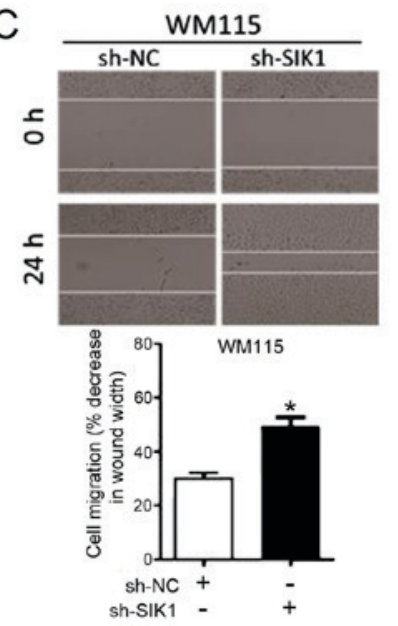

E

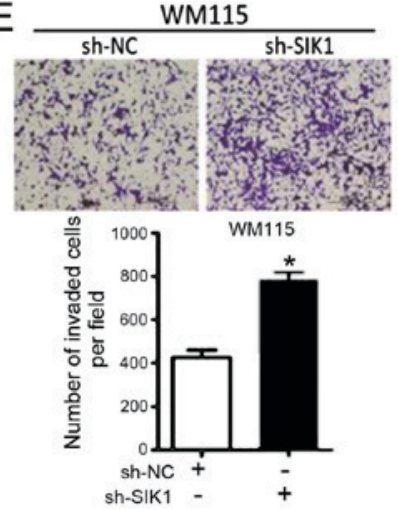

Figure 4. SIK1 promotes cell migration in melanoma. (A) Western blot analysis for SIK1 protein levels in A375 and WM115 cell lines after transfection with sh-SIK1 or sh-NC. A wound healing assay was performed after the transfection with sh-SIK1 or sh-NC in (B) A375 and (C) WM115 cells. A Transwell migration assay was performed after the transfection with sh-SIK1 or sh-NC in (D) A375 and (E) WM115 cells. ${ }^{*} \mathrm{P}<0.05$ vs sh-NC. SIK1, salt-inducible kinase 1; sh, short hairpin RNA; NC, negative control. Magnification, x100.

EMT, tumor growth and metastasis and provided a potential new candidate for HCC therapy (28). Reduced expression of SIK1 was also identified to be associated with poor outcomes in gastric carcinoma (19). Furthermore, SIK1 could be the potential target for numerous miRs. In particular, miR-203 induces proliferation, migration and invasion by targeting SIK1 in pancreatic cancer (29). In ovarian cancer, miR-141 promotes cell growth through reversing SIK1-suppressed proliferation and cancer stem cell-associated traits (30). However, the phenotypic role of SIK1 in melanoma remains largely unknown. In the present study, it was identified that downregulation of SIK1, as a target of miR-373, elevates cell migration of melanoma.

In summary, the present study identified that miR-373 functions as an oncomiR to promote melanoma progression through targeting SIK1 expression. This signaling pathway may provide a new therapeutic approach for melanoma.

\section{Acknowledgements}

Not applicable.

\section{Funding}

No funding was received.

\section{Availability of data and materials}

Not applicable.

\section{Authors' contributions}

$\mathrm{XB}$ performed the total experiments and was a major contributor in writing the manuscript. MY collected and analyzed the clinical data regarding the melanoma. YX contributed to the design of this project. All authors read and approved the final manuscript.

\section{Ethics approval and consent to participate}

The use of human tissues was approved by the Ethics Committee of the Central Hospital of Wuhan and all patients provided written, informed consent.

\section{Consent for publication}

Not applicable.

\section{Competing interests}

The authors declare that they have no competing interests.

\section{References}

1. Torre LA, Bray F, Siegel RL, Ferlay J, Lortet-Tieulent J and Jemal A: Global cancer statistics, 2012. CA Cancer J Clin 65: 87-108, 2015.

2. Cellerino P, Corsi F, Morandi E, Foschi D and Trabucchi E: Metastatic melanoma of the gallbladder. Eur J Surg Oncol 26: 815-816, 2000.

3. Haskaraca MF, Ozsoy M, Ozsan I and Kurt K: Primary malignant melanoma of the gallbladder: A case report and review of the literature. Case Rep Surg 2012: 693547, 2012.

4. Corrie P, Hategan M, Fife K and Parkinson C: Management of melanoma. Br Med Bull 111: 149-162, 2014.

5. Burki TK: Defining the genetics of melanoma progression. Lancet Oncol 17: e7, 2016

6. Zeng Y and Cullen BR: Sequence requirements for micro RNA processing and function in human cells. Rna 9: 112-123, 2003.

7. Bartel DP: MicroRNAs: Target recognition and regulatory functions. Cell 136: 215-233, 2009.

8. Li YC, Li CF, Chen LB, Li DD, Yang L, Jin JP and Zhang B: MicroRNA-766 targeting regulation of SOX6 expression promoted cell proliferation of human colorectal cancer. Onco Targets Ther 8: 2981-2988, 2015.

9. Cao J, Liu J, Xu R, Zhu X, Liu L and Zhao X: MicroRNA-21 stimulates epithelial-to-mesenchymal transition and tumorigenesis in clear cell renal cells. Mol Med Rep 13: 75-82, 2016.

10. Puglisi F, Minisini AM, De Angelis C and Arpino G: Overcoming treatment resistance in HER2-positive breast cancer: Potential strategies. Drugs 72: 1175-1193, 2012.

11. Bennett PE, Bemis L, Norris DA and Shellman YG: miR in melanoma development: miRNAs and acquired hallmarks of cancer in melanoma. Physiol Genomics 45: 1049-1059, 2013.

12. da Cruz AT and Jasiulionis MG: miRNAs and melanoma: How are they connected? Dermatol Res Pract 2012: 528345, 2012. 
13. Mannavola F, Tucci M, Felici C, Stucci S and Silvestris F: miRNAs in melanoma: A defined role in tumor progression and metastasis. Expert Rev Clin Immunol 12: 79-89, 2016.

14. Mueller DW, Rehli M and Bosserhoff AK: miRNA expression profiling in melanocytes and melanoma cell lines reveals miRNAs associated with formation and progression of malignant melanoma. J Invest Dermatol 129: 1740-1751, 2009.

15. Bertorello AM and Zhu JK: SIK1/SOS2 networks: Decoding sodium signals via calcium-responsive protein kinase pathways. Pflugers Arch 458: 613-619, 2009.

16. Lee J, Tong T, Takemori H and Jefcoate C: Stimulation of StAR expression by cAMP is controlled by inhibition of highly inducible SIK1 via CRTC2, a co-activator of CREB. Mol Cell Endocrinol 408: 80-89, 2015.

17. Cheng H, Liu P, Wang ZC, Zou L, Santiago S, Garbitt V, Gjoerup OV, Iglehart JD, Miron A, Richardson AL, et al: SIK1 couples LKB1 to p53-dependent anoikis and suppresses metastasis. Sci Signal 2: ra35, 2009.

18. Yao YH, Cui Y, Qiu XN, Zhang LZ, Zhang W, Li H and, Yu JM: Attenuated LKB1-SIK1 signaling promotes epithelial-mesenchymal transition and radioresistance of non-small cell lung cancer cells. Chin J Cancer 35: 50, 2016.

19. Selvik LK, Rao S, Steigedal TS, Haltbakk I, Misund K, Bruland T, Prestvik WS, Lægreid A and Thommesen L: Salt-inducible kinase 1 (SIK1) is induced by gastrin and inhibits migration of gastric adenocarcinoma cells. PLoS One 9: e112485, 2014.

20. Livak KJ and Schmittgen TD: Analysis of relative gene expression data using real-time quantitative PCR and the 2(-Delta Delta C(T)) method. Methods 25: 402-408, 2001.

21. Zhai W, Sun Y, Jiang M, Wang M, Gasiewicz TA, Zheng J and Chang C: Differential regulation of LncRNA-SARCC suppresses VHL-mutant RCC cell proliferation yet promotes VHL-normal RCC cell proliferation via modulating androgen receptor/HIF-2 $\alpha / \mathrm{C}-\mathrm{MYC}$ axis under hypoxia. Oncogene 35: 4866-4880, 2016.

22. Wei F, Cao C, Xu X and Wang J: Diverse functions of miR-373 in cancer. J Transl Med 13: 162, 2015.
23. Wei F, Wang Q, Su Q, Huang H, Luan J, Xu X and Wang J: miR-373 inhibits glioma cell U251 migration and invasion by down-regulating CD44 and TGFBR2. Cell Mol Neurobiol 36: 1389-1397, 2016

24. Zhang Y, Zhao FJ, Chen LL, Wang LQ, Nephew KP, Wu YL and Zhang S: MiR-373 targeting of the Rab22a oncogene suppresses tumor invasion and metastasis in ovarian cancer. Oncotarget 5: 12291-12303, 2014.

25. Eyking A, Reis H, Frank M, Gerken G, Schmid KW and Cario E: MiR-205 and MiR-373 are associated with aggressive human mucinous colorectal cancer. PLoS One 11: e0156871, 2016.

26. Ding W, Fan XL, Xu X, Huang JZ, Xu SH, Geng Q, Li R, Chen D and Yan GR: Epigenetic silencing of ITGA 2 by MiR-373 promotes cell migration in breast cancer. PLoS One 10: e0135128, 2015.

27. Chen D, Dang BL, Huang JZ, Chen M, Wu D, Xu ML, Li R and Yan GR: MiR-373 drives the epithelial-to-mesenchymal transition and metastasis via the miR-373-TXNIP-HIF1 $\alpha$-TWIST signaling axis in breast cancer. Oncotarget 6: 32701-32712, 2015

28. Qu C, He D, Lu X, Dong L, Zhu Y, Zhao Q, Jiang X, Chang P, Jiang X, Wang L, et al: Salt-inducible kinase (SIK1) regulates HCC progression and WNT/ $\beta$-catenin activation. J Hepatol 64: 1076-1089, 2016.

29. Ren ZG, Dong SX, Han P and Qi J: miR-203 promotes proliferation, migration and invasion by degrading SIK1 in pancreatic cancer. Oncol Rep 35: 1365-1374, 2016.

30. Chen JL, Chen F, Zhang TT and Liu NF: Suppression of SIK1 by miR-141 in human ovarian cancer cell lines and tissues. Int J Mol Med 37: 1601-1610, 2016. 